\title{
Toward early diagnosis in arrhythmogenic right ventricular dysplasia/cardiomyopathy
}

\author{
Richard N. W. Hauer
}

Received: 3 June 2009 / Accepted: 4 June 2009 /Published online: 15 August 2009

(C) Springer Science + Business Media, LLC 2009

Arrhythmogenic right ventricular dysplasia/cardiomyopathy $(\mathrm{ARVD} / \mathrm{C})$ is a myocardial disease which is histologically mainly localized in the right ventricle (RV) [1-3]. Histology shows fibrofatty replacement of the myocardium. At autopsy, diagnosis is usually straightforward. In the clinical setting, tissue is obtained by RV endomyocardial biopsies. For safety reasons, sampling is usually carried out from the interventricular septum to avoid perforation of the affected thin RV free wall. However, histologic diagnosis by endomyocardial biopsy is hampered by (1) the often focal nature of the disease, (2) only subendocardial tissue sampling, and (3) interventricular septum rarely involved in the disease process.

Since the histologic yield of septal biopsies is low, other diagnostic criteria were needed. A set of criteria was proposed by the Task Force of the European Society of Cardiology and the Scientific Council on Cardiomyopathies of the International Society and Federation of Cardiology in 1994 [4]. These Task Force criteria are universally used and facilitate comparison of results of scientific and clinical studies. These criteria are very specific but often lack sensitivity, necessitating identification of additional criteria [5]. New Task Force criteria are expected in the near future. Both previous and new Task Force criteria are based on family history, depolarization and repolarization abnormalities, arrhythmias, and also structural disease, including histology.

On the other hand, histologic yield may improve if sampling is carried out carefully from the RV free wall, preferably guided by electro-anatomic voltage mapping [6,

R. N. W. Hauer $(\bowtie)$

Department of Cardiology, University Medical Center,

Utrecht, The Netherlands

e-mail: r.n.w.hauer@umcutrecht.nl
7]. Endomyocardial biopsies were obtained from lowvoltage areas, preferably from the border zone to minimize the risk of perforation. Nevertheless, the risk of perforation is still not negligible since pericardial effusion, although minimal, was observed in two of 22 patients [6]. In addition, the limitation of only sampling subendocardial tissue remains-since in early stages of the disease, histopathologic changes may be limited to subepicardial and midmyocardial layers.

In recent years, $\mathrm{ARVD} / \mathrm{C}$ appeared to be often a genetic disease, with pathogenic mutations encoding desmosomal proteins [8-12]. Desmosomes are structures in the intercalated disk involved in mechanical coupling of adjacent myocardial cells. Mutations in desmosomal genes alter the number or integrity of desmosomes [13]. This may be followed by connexin 43 remodeling in the gap junctions $[14,15]$. This process will give rise to electrical uncoupling of adjacent myocardial cells, which contributes to activation delay and arrhythmogenesis. Changes in the various intercalated disk proteins are detectable with immunohistochemical staining. Because of the molecular genetic background of $\mathrm{ARVD} / \mathrm{C}$ and some preliminary findings, we hypothesized that remodeling of the intercalated disks may be found not only in the RV but also in left ventricle and interventricular septum [16].

Asimaki et al. [17] performed immunohistochemistry for desmosomal proteins in three groups of patients. In the first group, myocardial samples were obtained on autopsy $(n=9)$ or endomyocardial biopsy $(n=2)$. Eight of these patients were known with a desmosomal gene mutation. All patients showed reduced desmosomal plakoglobin, whereas this was not observed in control samples. In most patients, levels of desmoplakin and plakophilin-2 were also reduced. The second group consisted of 15 patients, five subjects each with hypertrophic, dilated, or ischemic heart disease. All 
had normal levels of plakoglobin in the intercalated disk. The usefulness of immunohistochemistry analysis was proven in the third group consisting of 11 patients with proven ARVD/C, 14 with probable ARVD/C, and five nonARVD/C. A reduced level of plakoglobin showed a sensitivity of $91 \%$, specificity of $82 \%$, positive predictive value of $83 \%$, and negative predictive value of $90 \%$. Plakoglobin was also reduced at the intercalated disk in normal-appearing regions in the left ventricle and interventricular septum. Further studies are needed for evaluation of other intercalated disk proteins in different mutation groups.

The study by Asimaki is of practical importance since it suggests that immunohistochemical analysis can be carried out from endomyocardial samples originating from the right side of the interventricular septum, which is relatively safe. The presence of immunohistochemical abnormalities in the absence of histologic pathology suggests that immunohistochemical alterations precede histologic abnormality. Thus, immunohistochemical analysis may facilitate ARVD/C diagnosis in an early stage of disease.

\section{References}

1. Marcus, F. I., Fontaine, G. H., Guiraudon, G., Frank, R., Laurenceau, J. L., Malergue, C., et al. (1982). Right ventricular dysplasia: A report of 24 adult cases. Circulation, 65, 384-398.

2. Corrado, D., Basso, C., Thiene, G., McKenna, W. J., Davies, M. J., Fontaliran, F., et al. (1997). Spectrum of clinicopathologic manifestations of arrhythmogenic right ventricular cardiomyopathy/dysplasia: A multicenter study. J Am Coll Cardiol, 30, 1512-1520.

3. Basso, C., Thiene, G., Corrado, D., Angelini, A., Nava, A., \& Valente, M. (1996). Arrhythmogenic right ventricular cardiomyopathy: Dysplasia, dystrophy, or myocarditis? Circulation, 94, 983-991.

4. McKenna, W. J., Thiene, G., Nava, A., Fontaliran, F., BlomstromLundqvist, C., Fontaine, G., et al. (1994). Diagnosis of arrhythmogenic right ventricular dysplasia/cardiomyopathy. Task Force of the Working Group Myocardial and Pericardial Disease of the European Society of Cardiology and of the Scientific Council on Cardiomyopathies of the International Society and Federation of Cardiology. Br Heart J, 71, 215-218.

5. Cox, M. G. P. J., Nelen, M. R., Wilde, A. A. M., Wiesfeld, A. C., Vd Smagt, J., Loh, P., et al. (2008). Activation delay and VT parameters in arrhythmogenic right ventricular dysplasia/cardiomyopathy: Toward improvement of diagnostic ECG criteria. $J$ Cardiovasc Electrophysiol, 19, 775-781.
6. Avella, A., d'Amati, G., Pappalardo, A., Re, F., Silenzi, P. F., Laurenzi, F., et al. (2008). Diagnostic value of endomyocardial biopsy guided by electroanatomic voltage mapping in arrhythmogenic right ventricular cardiomyopathy/dysplasia. J Cardiovasc Electrophysiol, 19, 1127-1134.

7. Corrado, D., Basso, C., Leoni, L., Tokajuk, B., Bauce, B., Frigo, G., et al. (2005). Three-dimensional electroanatomic voltage mapping increases accuracy of diagnosing arrhythmogenic right ventricular cardiomyopathy/dysplasia. Circulation, 111, 30423050 .

8. McKoy, G., Protonotarios, N., Crosby, A., Tsatsopoulou, A., Anastasakis, A., Coonar, A., et al. (2000). Identification of a deletion in plakoglobin in arrhythmogenic right ventricular cardiomyopathy with palmoplantar keratoderma and woolly hair (Naxos disease). Lancet, 355, 2119-2124.

9. Rampazzo, A., Nava, A., Malacrida, S., Beffagna, G., Bauce, B., Rossi, V., et al. (2002). Mutation in human desmoplakin domain binding to plakoglobin causes a dominant form of arrhythmogenic right ventricular cardiomyopathy. American Journal of Human Genetics, 71, 1200-1206.

10. Gerull, B., Heuser, A., Wichter, T., Paul, M., Basson, C. T., McDermott, D. A., et al. (2004). Mutations in the desmosomal protein plakophilin-2 are common in arrhythmogenic right ventricular cardiomyopathy. Nature Genetics, 36, 1162-1164.

11. Pilichou, K., Nava, A., Basso, C., Beffagna, G., Bauce, B., Lorenzon, A., et al. (2006). Mutations in desmoglein-2 gene are associated with arrhythmogenic right ventricular cardiomyopathy. Circulation, 113, 1171-1179.

12. Syrris, P., Ward, D., Evans, A., Asimaki, A., Gandjbakhch, E., Sen-Chowdhry, S., et al. (2006). Arrhythmogenic right ventricular dysplasia/cardiomyopathy associated with mutations in the desmosomal gene desmocollin-2. American Journal of Human Genetics, 79, 978-984.

13. Basso, C., Czarnowska, E., Della Barbera, M., Bauce, B., Beffagna, G., Wlodarska, E. K., et al. (2006). Ultrastructural evidence of intercalated disc remodelling in arrhythmogenic right ventricular cardiomyopathy: An electron microscopy investigation on endomyocardial biopsies. European Heart Journal, 27, 18471854.

14. Saffitz, J. E. (2005). Dependence of electrical coupling on mechanical coupling in cardiac myocytes: Insights gained from cardiomyopathies caused by defects in cell-cell connections. Annals of the New York Academy of Sciences, 1047, 336-344.

15. Oxford, E. M., Musa, H., Maass, K., Coombs, W., Taffet, S. M., \& Delmar, M. (2007). Connexin43 remodeling caused by inhibition of plakophilin-2 expression in cardiac cells. Circ Res, 101(7), 703-711.

16. Hauer, R. N. W., \& Cox, M. G. P. J. (2008). The challenge of early diagnosis in arrhythmogenic right ventricular dysplasia/ cardiomyopathy. J Cardiovasc Electrophysiol, 19, 1135-1136.

17. Asimaki, A., Tandri, H., Huang, H., Halushka, M. K., Gautam, S., Basso, C., et al. (2009). A new diagnostic test for arrhythmogenic right ventricular cardiomyopathy. N Engl J Med, 360, 1075-1084. 